\title{
Research on the Control Method of External Characteristic of Speed Based on CVT
}

\author{
Xunsong Yan \\ Changchun Institute of Engineering and Technology, \\ Changchun, Jilin \\ e-mail:47335774e@qq.com
}

\begin{abstract}
According to the changes of the traditional speed ratio control of CVT speed ratio frequently, resulting in low efficiency, serious wear of CVT, researched a method of speed ratio control based on external characteristic of engine, to ensure the change speed ratio of CVT economy and power performance. The theoretical study and simulation analysis results indicate that the proposed speed ratio control method in the best economic area can effectively reduce the frequency of speed ratio regulation of CVT, thus improve CVT efficiency and service life.
\end{abstract}

Keywords-component; CVT, control method, External characteristic

\section{INTRODUCTION}

Speed ratio control of general CVT was performed according to the best economic engine characteristic curve fitting curve or optimal power curve. Continuous variable speed, will make the CVT active bevel gear and driven bevel gear under the effect of the hydraulic cylinder to do the axial movement. With CVT metal from the wheel slip in the main, CVT, resulting in reduced efficiency, at the same time, accelerate the metal belt wear and bevel wheel. In order to solve this problem, improve the transmission efficiency of CVT, CVT speed ratio control method is developed in this paper which based on external characteristic of engine. In the premise of ensuring the power and economy under control, the method can reduce the CVT of metal belt slipping, improve transmission efficiency and service life of CVT.

\section{The Traditional CVT SpeEd RAtio Control}

The key of CVT speed ratio control is to determine the target speed follow control problem of target speed and actual speed ratio. The definition of the CVT speed ratio transmission device for driving wheel speed and driven wheel speed ratio, i.e.

$$
i=\frac{n_{d r}}{n_{\text {out }}}=\frac{n_{e}}{n_{\text {out }}}
$$

$n_{d r}$ :driving wheel speed; $n_{\text {out }}$ driven wheel speed; $n_{e}$ engine speed.

Therefore, the definition of CVT target speed ratio is engine target speed $n_{e o}$ divided by driven wheel target speed $^{n_{\text {out }}}$ :

$$
\begin{aligned}
& \text { Xiaoyu Wang } \\
& \text { Changchun Institute of Engineering and Technology, } \\
& \text { Changchun, Jilin } \\
& \text { e-mail: 14662375e@qq.com } \\
& i_{o}=\left\{\begin{array}{cc}
i_{\max } & n_{\varepsilon o} / n_{o u t} \geq i_{\max } \\
n_{\varepsilon o} / n_{\text {out }} ; i_{\min } \leq n_{\varepsilon o} / n_{o u t} \leq i_{\max } \\
i_{\min } & n_{\varepsilon o} / n_{\text {out }} \leq i_{\max }
\end{array}\right.
\end{aligned}
$$

From equation (2) can be seen, the key of target speed ratio is to determine target engine speed, after determining target engine speed, CVT target speed ratio is determined. According to the engine universal characteristics of engine the best economic performance curve and the best power characteristic curve can determine the target engine speed. A universal characteristic of engine and the best power characteristic curve and the best economic performance curve as shown in figure 1.

Best economic performance curve

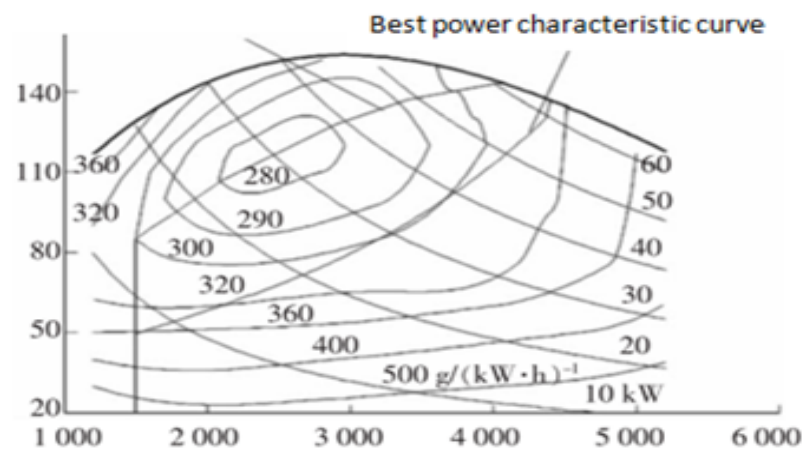

Figure 1. Engine universal characteristic curve

In practice, to make the engine work in the lowest fuel consumption of the engine speed, need engine work on the minimum fuel consumption condition, at that time the engine throttle opening and engine speed work according to the best economic speed characteristic curve of engine. The continuous change of throttle opening, engine has best power characteristic curve and the best economic performance curve as shown in figure 2 . 


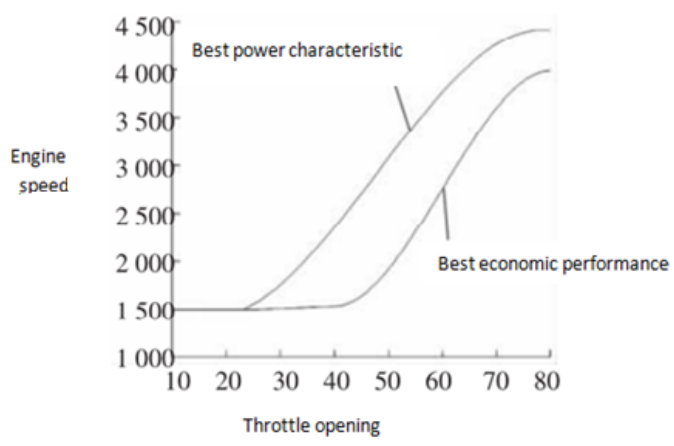

Figure 2. Speed characteristic curve of engine

According to best power characteristic curve and the best economic performance curve can determine the rotation speed of the engine work in the best power line and the best economic line at a throttle valve opening, i.e. formula (2) the target engine speed. First of all, the target engine speed which is determined by the best economic performance curve, is stored in a memory controller. A driven bevel wheel is collected speed by the wheel speed sensor real-time acquisition, according to formula (2) to determine the target speed ratio. CVTECU can complete stepless speed change, and control the actual speed ratio and the target speed ratio.

\section{RESEARCH ON THE BEST ECONOMIC AREA OF SPEED RATIO CONTROL}

In the light of the the defects of traditional CVT speed ratio control, considering the actual engine working, in the wide range of the best economic performance curve,, the actual engine fuel consumption rate of change is very small, the engine characteristic curve as shown in Figure 3 and Figure

4 shows.

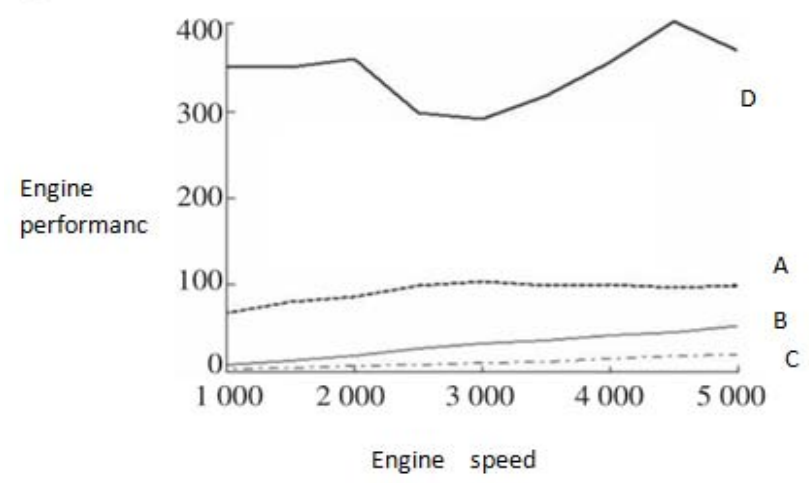

Figure 3. Engine external characteristic test

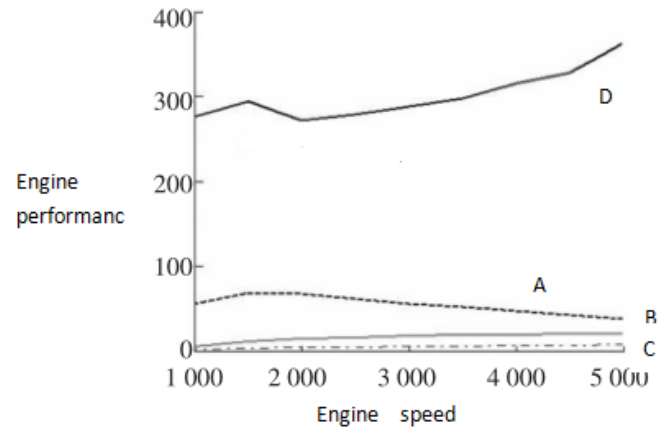

Figure 4. Engine throttle valve opening 20\% speed characteristics test

A: effective torque, B: effective power, C: fuel consumption, D: fuel consumption rate.

We can see from Figure 3, the best fuel economy speed is in range of $2500-3500 \mathrm{r} / \mathrm{min}$, the variation rate of fuel consumption is in the $10-30 \mathrm{~g} / \mathrm{kw}$..h, the best power characteristic speed is in range of $3000-4000 \mathrm{r} / \mathrm{min}$, the maximum effective torque change amount is in range of 0 $5 \mathrm{~N} \cdot \mathrm{m}$. The experimental data show that, in a wide range of engine speed changes, changes in the best fuel economic performance and the best power characteristic is very small, the vehicle fuel economy and power influence is very small. Similarly, we can see from Figure 4, in the speed range of $2500-3500 \mathrm{r} / \mathrm{min}$, the variation of torque and fuel consumption rate of the variation is very small. Therefore, the speed ratio control in CVT, can consider to delay the target speed ratio control, which could not change the CVT speed target ratio control in the best fuel economy speed and best power characteristic speed range. In the engine speed, the driver can change the accelerating pedal to achieve the intention or increase the torque by adjusting the engine itself to complete.

By the principle of engine shows that the engine has torque and rotational speed regulation ability, in order to adapt to the external power, or when the vehicle is running do not need to shift in a certain range, the engine can be adjusted automatically, reduce speed increasing torque to overcome the resistance to the vehicle. When the external force is reduced to automatically adjust the speed increase, the equivalent of the engine has continuously variable transmission capability of the torque and speed range. The torque and speed adaptation ability to adapt, usually represented by the coefficient of torque and rotational speed adaptive coefficient adaptive $K_{T}, K_{n}$.

$$
\begin{gathered}
K_{T}=\frac{T_{t q \max }}{T_{\text {tqn }}} \\
K_{n}=\frac{n_{n}}{n_{m}}
\end{gathered}
$$

In the formula, $T_{\text {tq } \max }$ :the maximum engine torque; $T_{\text {tan }}$ :rated torque and speed; ${ }_{n}$ :rated speed; ${ }^{n}$ :rated torque.

The coefficient of torque adaptability of general gasoline engine is $K_{T}$, which is about 1.25-1.35, the coefficient of speed adaptive is $K_{n}$, which is about 1.6-2.5. It is mean that 
when the torque is $1.25-1.35$ times, speed is 1.6-2.5 times, engine can be adjust the speed and torque in order to adapt to the changes in the external conditions without the intervention of transmission, and achieve continuously variable transmission characteristics in small range. The fundamental reason is actually a stepped automatic transmission has accelerated the ability on each gear range.

Based on this principle, we researched on the control method of external characteristic of speed based on CVT. Considering the above factors, regulation ability in the actual speed ratio control is give full play to its own engine, make the target speed ratio control in best economic area, as shown in figure 5.

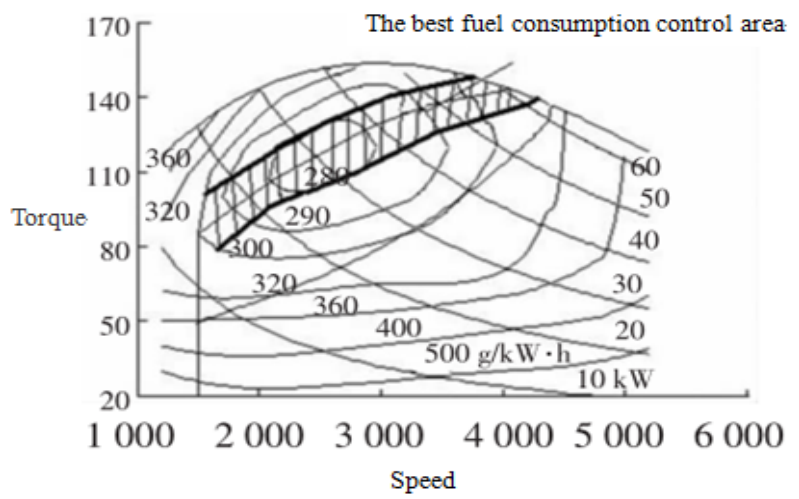

Figure 5. The engine target speed control

\section{SimUlation AnAlysis}

Researched on the control method of speed based on CVT, use AVL-CRUISE and MATLAB-SIMULINK simulation platform, built CVT forward simulation model, analysis the influence of speed ratio control strategy on vehicle performance.

CRUISE is a modular, parameterized modeling software, according to the structure of the vehicle, drag the corresponding component module, input module parameters, such as the vehicle model to engine MAP data, CVT data, build CVT model. CRUISE vehicle model consists of vehicle model, engine model, CVT model, rear axle model, brake model, tire model and so on. In the simulation, the CRUISE vehicle model with CVT control prototype model, the speed ratio control of CVT is through the Matlab-DLL CRUISE module in the vehicle model, the speed ratio control strategy model of dynamic link library file calls generated by the SIMULINK build, CVT speed ratio control is implemented.

SIMULINK speed ratio control strategy is mainly composed of a control parameter input module, Speed ratio control module and a control parameters of the output module. The control parameter input module is mainly used to input the basic parameters required by the CVT speed ratio control, such as engine speed, driving and driven bevel wheel speed, acceleration pedal, brake pedal. According to the simulation theory, the CRUISE vehicle model was the prototype for simulation and control, so the control strategy of input control parameters were derived from the CRUISE vehicle model. The control parameters of the output module output control CVT ratio change target speed ratio, engine target speed and target torque, target accelerator pedal opening degree, the target speed ratio is the key.

The most importance method of CVT speed ratio control is the target speed ratio control module, curve of target speed ratio compared to the conventional speed control, the target speed ratio is the best economic area ratio. In the control, the changes of the target ratio is not continuous, but according to the engine speed and the vehicle driving power demand of the target ratio, make the engine work in the best economic area, play engine adaptive characteristics. When the engine is in the best economic area of the regulation cannot meet the conditions of power demand, change the target ratio to make the engine work in the best economic area. In this paper, the simulation of the car driving cycle simulation analysis comparison to have the representative typical working condition of NEDC in Europe, as shown in figure 6.

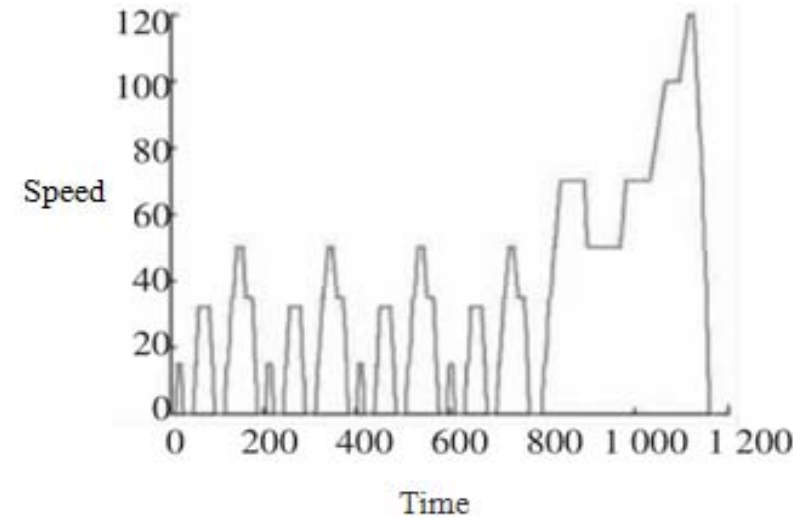

Figure 6. NEDC cycle simulation

The co-simulation of NEDC conditions, a CVT target speed ratio control and traditional CVT control simulation curve as shown in figure 7.

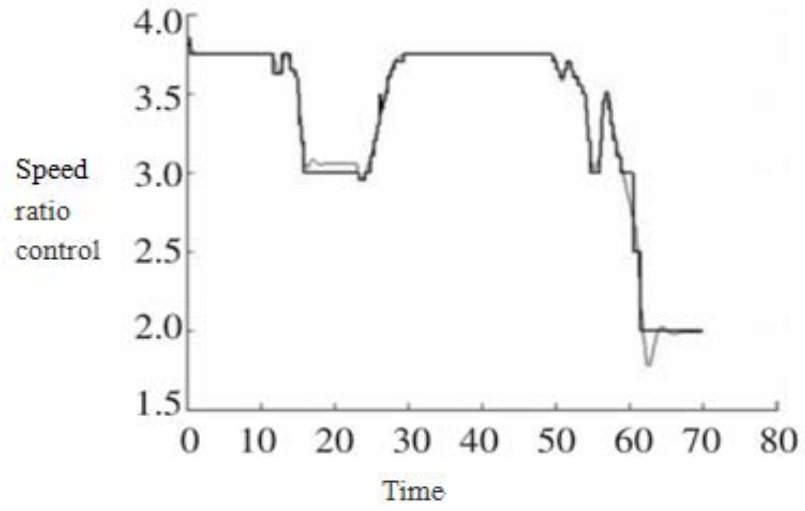

Figure 7

As can be seen from Figure 7, in comparison with the change of traditional target ratio, the best economic area ratio, in some period of time, because of best economic area is limit by the engine speed ratio range (such as engine 
speed in the economic area, the ratio can be kept constant), and target speed ratio change of traditional speed control follow the best engine economic curve, speed ratio is continuous adjustment, frequent changes. From two kinds of fuel economy of the speed ratio control, NEDC cycle fuel economy are best economic area ratio control for 7.89L/100 $\mathrm{km}$, the best economic curve control8.01L/100km, best economic area ratio control fuel consumption decreased slightly.

Therefore, the control method of best economic area ratio, at the same time improve the fuel economy of reduced frequency change ratio of CVT, reduced the number of driving wheel cylinder, reduced the metal belt slipping in the number of cone wheel, thereby reduced the metal belt and grinding wheel sliding cone.

\section{ACKNOWLEDGMENT}

The preferred spelling of the word "acknowledgment" in America is without an "e" after the "g". Avoid the stilted expression, "One of us (R.B.G.) thanks." Instead, try "R.B.G. thanks". Put applicable sponsor acknowledgments here; DO NOT place them on the first page of your paper or as a footnote.

\section{REFERENCES}

[1] Y.k. Zhang et al. Study on the design method of the CVT control system of metal V-belt type. Automobile technology, 2004 (10): 7 10

[2] Y.H Lu et al. Design of Infenion C164CI control system based on the metal belt type CVT. Journal of Jilin University (Engineering and Technology Edition).2006,36:84 88 